\title{
An effective methodology to design scale model of non-metallic structural entity
}

\author{
Liming Yuan ${ }^{\text {a }}$, Zhijie Xie, Fei Dai, Yonggang Xu, Yuan Zhang \\ Science and Technology on Electromagnetic Scattering Laboratory, Shanghai 200438, China
}

\begin{abstract}
In order to solve the problem on designing scale model of non-metallic structural entity, a method is first proposed according to the high-frequency approximation algorithm, in which reflection coefficients of designed scale model are optimized to be as identical as possible with those of theoretical scale model. An example is given to verify the effectiveness of the method, where a non-metallic cone is constructed and FEKO is employed to simulate the monostatic radar Cross Section (RCS) of the designed scale cone and the theoretical scale cone. Result reveals that the monostatic RCS of the designed scale cone agrees very well with that of the theoretical scale cone. The mean deviation of the monostatic RCS is just $1.31 \mathrm{~dB}$ in simulated elevation angles of $0^{\circ} \sim 90^{\circ}$. Thus, the method proposed in this paper is of important theoretical and practical significance in constructing scale model of high accuracy.
\end{abstract}

\section{Introduction}

Scale measurement technology has very important significance in studying electromagnetic scattering properties ${ }^{[1,2]}$. In scale measurement, one of keys is to construct an accurate scale model. Existing researches begin with studying scale models of perfect electric conductor (PEC) and then come to study scale models of non-metallic materials as more and more composites are applied to objects ${ }^{[3-7]}$. However, the non-metallic materials in the existing studies have plated-shape without special circumstances. The non-metallic materials are always mounted on object surface and the scale materials can be designed according to the reflective loss. As the development of manufacturing technologies of composites, more and more non-metallic structural entities could be found in objects such as vehicles, aircraft, ships and so on. These non-metallic entities have great influences on the scattering properties. Meanwhile, these non-metallic entities not only have complicated electromagnetic properties but also have special geometric shapes. As it is always very difficult to manufacture scale material which has the same electromagnetic parameters with theoretical scale material, there is hardly any solution to solve the problem on constructing scale model of non-metallic structural entity over years. In this paper, a method is first proposed to solve the problem according to the high-frequency approximation algorithm. In order to verify the effectiveness of the designed result by the method, a nonmetallic cone is constructed and FEKO is employed to simulate the monostatic radar Cross Section (RCS) of the designed scale cone and the theoretical scale cone.

\section{Theories and methods}

According to the high-frequency approximation algorithm, the scattering field can be calculated by the Stratton-Chu formula ${ }^{[8]}$, which is expressed as follows,

$$
\begin{gathered}
\mathbf{E}^{s}=\frac{j k}{4 \pi} \frac{e^{-j k r}}{r} \int_{s} \hat{s} \times\left(\mathbf{M}_{s}+\eta_{0} \hat{s} \times \mathbf{J}_{s}\right) e^{j k r^{\prime}(\hat{s}-\hat{i})} d s^{\prime} \\
\mathbf{H}^{s}=-\frac{j k}{4 \pi} \frac{e^{-j k r}}{r} \int_{s} \hat{s} \times\left(\mathbf{J}_{s}-\frac{1}{\eta_{0}} \hat{s} \times \mathbf{M}_{s}\right) e^{j k r^{\prime}(\hat{s}-\hat{i})} d s^{\prime}
\end{gathered}
$$

where $\mathbf{E}^{s}$ and $\mathbf{H}^{\mathrm{s}}$ are scattering electric and magnetic field, respectively; $\mathbf{M}_{s}$ and $\mathbf{J}_{s}$ are induced electric and magnetic current on the surface, respectively; $\eta_{0}$ is the impedance in free space; $k$ is the propagation constant in free space; $r$ is the distance; $\hat{s}$ and $\hat{i}$ are unit vectors in scattering direction and incident direction, respectively; $S$ is the entire surface of target. The induced electric current and magnetic current are calculated by the following expressions,

$$
\begin{aligned}
& \mathbf{J}_{s}= {\left[-\left(1-\Gamma_{\perp}\right)\left(\hat{\mathbf{e}}_{\perp} \cdot \hat{\mathbf{e}}_{\mathbf{i}}\right)(\hat{\mathbf{i}} \cdot \hat{\mathbf{n}}) \hat{\mathbf{e}}_{\perp} E^{i}+\left(1+\Gamma_{\square}\right)\left(\hat{\mathbf{e}}_{\square} \cdot \hat{\mathbf{e}}_{\mathbf{i}}\right) \hat{\mathbf{n}} \times \hat{\mathbf{e}}_{\perp} E^{i}\right] / \eta_{0} } \\
& \mathbf{M}_{s}=\left(1+\Gamma_{\perp}\right)\left(\hat{\mathbf{e}}_{\perp} \cdot \hat{\mathbf{e}}_{\mathbf{i}}\right) \hat{\mathbf{n}} \times \hat{\mathbf{e}}_{\perp} E^{i}+\left(1-\Gamma_{\square}\right)\left(\hat{\mathbf{e}}_{\square} \cdot \hat{\mathbf{e}}_{\mathbf{i}}\right)(\hat{\mathbf{n}} \cdot \hat{\mathbf{i}}) \hat{\mathbf{e}}_{\perp} E^{i}
\end{aligned}
$$

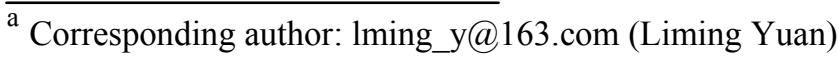


in which, $\hat{\mathbf{n}}, \hat{\mathbf{i}}$ and $\hat{\mathbf{s}}$ are unit vectors in normal direction of surface, incident direction and scattering direction, respectively; $\hat{\mathbf{e}}_{\mathbf{i}}$ is the unit vector of incident electric field; $\hat{\mathbf{e}}_{\perp}$ and $\hat{\mathbf{e}}_{\square}$ are unit vectors of incident electric field in incident plane and perpendicular to the incident plane; $\Gamma_{\perp}$ and $\Gamma_{\square}$ denote reflection coefficients irradiated by TM and TE wave, respectively. It can be seen that the same reflection coefficients lead to the same induced electric and magnetic current and then the same scattering property. When scale material is designed by reflection coefficients, both $\Gamma_{\perp}$ and $\Gamma_{\square}$ should be taken into account.

They can be calculated by following expressions,

$$
\begin{gathered}
\Gamma_{\perp}=\frac{\cos \theta_{i}-\sqrt{\varepsilon_{r} \mu_{r}-\sin ^{2} \theta_{i}}}{\cos \theta_{i}+\sqrt{\varepsilon_{r} \mu_{r}-\sin ^{2} \theta_{i}}} \\
\Gamma_{\square}=\frac{\varepsilon_{r} \mu_{r} \cos \theta_{i}-\sqrt{\varepsilon_{r} \mu_{r}-\sin ^{2} \theta_{i}}}{\varepsilon_{r} \mu_{r} \cos \theta_{i}+\sqrt{\varepsilon_{r} \mu_{r}-\sin ^{2} \theta_{i}}}
\end{gathered}
$$

in which, $\theta_{i}$ is incident angle; $\varepsilon_{r}$ and $\mu_{r}$ are relative permittivity and permeability of material. According to Eq. (3a) and (3b), scale model of non-metallic entity can be designed by following four steps.

(i) Input reflection coefficients of theoretical scale material. The reflection coefficients are calculated at different incident angles.

(ii) Built a collection of electromagnetic parameters at scale frequency. Selected some inclusions and mix them with a binder in different proportions. Measure electromagnetic parameters of mixtures and fit the measured data by effective medium theories. Built the collection of electromagnetic parameters by the fitting formulae and number each pair of permittivity and permeability.

(iii) Perform loop calculations. In each loop calculation, permittivity and permittivity are determined by a number generated by a random function. Calculate reflection coefficients and deviation by comparing with the reflection coefficients of theoretical scale material. Record the deviation and the corresponding electromagnetic parameters.

(iv) Search the optimal result. After the loop calculations, find out the optimal result by the minimum deviation of calculated reflection coefficients. The scale material could be determined according to the record in step (ii) and the designed scale model has the same geometric parameters with the theoretical scale model.

\section{Results and discussions}

Now an example is given to verify the effectiveness of the method. It is hypothesized that a non-metallic material is non-magnetic and its permittivity is $1-100 \mathrm{j}$. The measurement frequency in full size system is $10 \mathrm{GHz}$ and then the corresponding frequency in scale system is $100 \mathrm{GHz}$ as the scale ratio is $1 / 10$. The scale material can be designed by the method introduced in section 2 . Here, the collection of electromagnetic parameters at $100 \mathrm{GHz}$ is built by random functions. The collection includes 1000 pairs of permittivity $\left(\varepsilon_{r}=\varepsilon_{r}^{\prime}-j \varepsilon_{r}^{\prime \prime}\right)$ and permeability $\left(\mu_{r}=\mu_{r}^{\prime}-j \mu_{r}^{\prime \prime}\right)$. All $\varepsilon_{r}^{\prime}, \varepsilon_{r}^{\prime \prime}, \mu_{r}^{\prime}$ and $\mu_{r}^{\prime \prime}$ are in $1 \sim 50,0 \sim 5$, $1 \sim 5$ and $1 \sim 2$, respectively. Record and number each pair of permittivity and permeability. After optimizing, the permittivity and permeability of designed scale material are $47.66-1.86 \mathrm{j}$ and $0.29-1.89 \mathrm{j}$, respectively. Figure 1 plots the complex reflection coefficients of the designed scale material and the theoretical scale material irradiated by TM wave and TE wave as the incidence angle changes from $0^{\circ}$ to $90^{\circ}$. As shown in Fig. 1, it can be seen that the reflection coefficients of the designed scale material are very identical with those of the theoretical scale material.
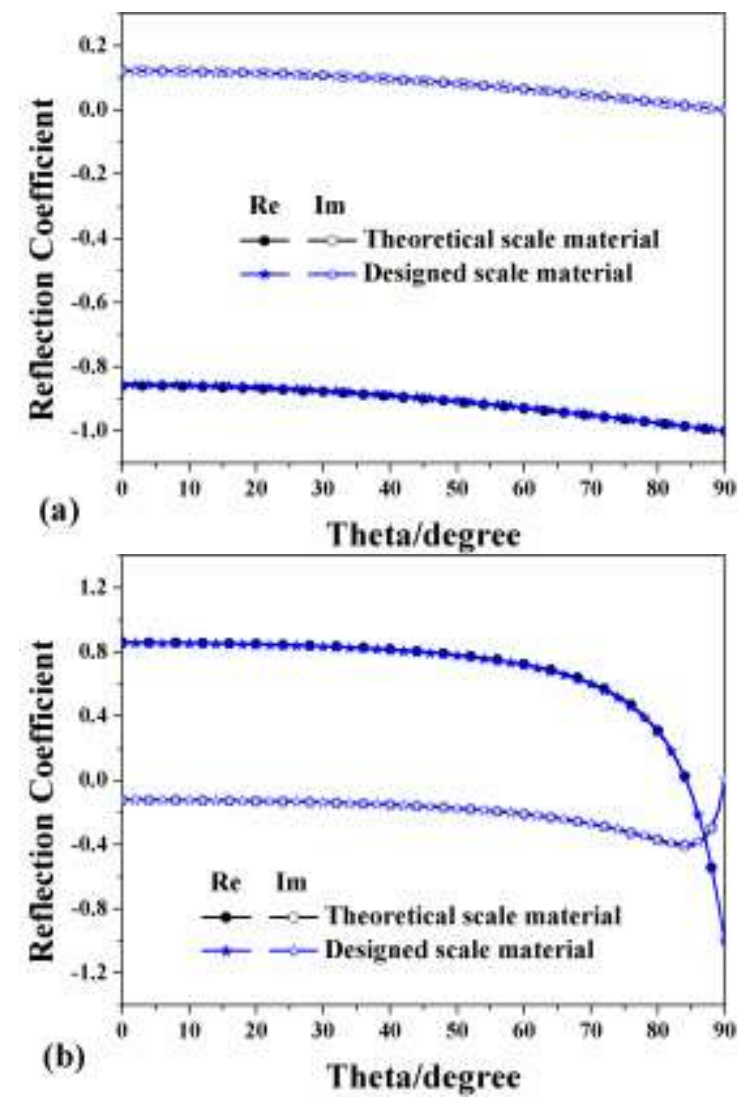

Fig. 1 The complex reflection coefficients of the theoretical scale material and the designed scale material irradiated by TM wave (a) and TE wave (b).

As introduced in section 2, designed scale model of non-metallic structural entity has the same geometric parameters with theoretical scale model. Next, a nonmetallic cone is constructed and its monostatic RCS is simulated by commercial software FEKO. The nonmetallic cone has a height of $500 \mathrm{~mm}$ and a PEC bottom of a $1000 \mathrm{~mm}$ diameter. The cone is comprised of nonmagnetic material and the permittivity except the bottom is $1-100 \mathrm{j}$. The designed scale cone and the theoretical scale cone have the same geometric parameters which are $1 / 10$ of the corresponding parameters of the full size cone. The electromagnetic parameters of the theoretical scale cone are the same with those of the full size cone and the designed scale cone has the permittivity of 47.66-1.86j and the permeability of $0.29-1.89 \mathrm{j}$. In simulations, monostatic RCS is calculated; TE wave is selected; the

\footnotetext{
a Corresponding author: lming_y@163.com (Liming Yuan)
} 
frequency is $100 \mathrm{GHz}$; the azimuth angle is $0^{\circ}$ and the elevation angle changes from $0^{\circ}$ to $90^{\circ}$.

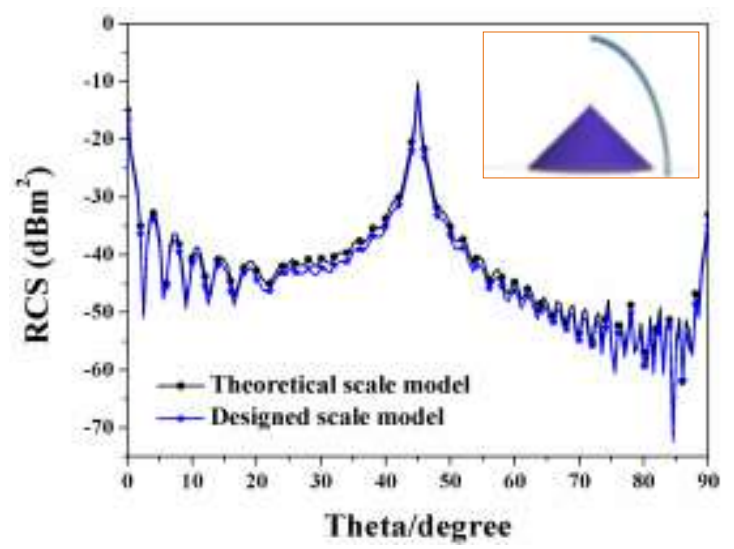

Fig. 2 The simulated RCS of the designed scale model and the theoretical scale model of the non-metallic cone.

Figure 2 shows the simulated RCS of the designed scale model and the theoretical scale model of the nonmetallic cone. As shown in the figure, it can be seen that there is a good agreement on monostatic RCS between the designed scale cone and the theoretical scale cone. The average value of absolute deviations is just $1.31 \mathrm{~dB}$ in $0^{\circ} \sim 90^{\circ}$. And it is $1.20 \mathrm{~dB}, 1.42 \mathrm{~dB}$ and $1.31 \mathrm{~dB}$ in $0^{\circ} \sim 30^{\circ}, \quad 30^{\circ} \sim 60^{\circ}$ and $60^{\circ} \sim 90^{\circ}$, respectively. The deviation mainly comes from non-plane scattering sources but it is completely in the range of RCS measurement tolerance.

\section{Conclusions}

According to the high-frequency approximation algorithm, a method is first proposed to design scale model of non-metallic structural entity. In order to verify the effectiveness of the method, an example is given to design scale model of a non-metallic cone. The simulated
RCS of the designed scale cone is compared with that of the theoretical scale cone and the average value of absolute deviations is just $1.31 \mathrm{~dB}$ in $0^{\circ} \sim 90^{\circ}$. The deviation completely falls within the RCS measurement tolerance. Therefore, it can be concluded that the method proposed in this paper is very effective to design scale model of non-metallic structural entity.

\section{Acknowledgements}

This work was supported by Shanghai Municipal Science and Technology Commission under Grant Nos.18ZR1437400，17YF1418400，17QB1404100, 17210730900 and 16XD1423300, and the National Civil Aircraft Project of China under Grant MJZ-2017-F-11.

\section{References}

[1] White M O 1998 Electronics \& communication engineering journal 10169

[2] Zhao Y et al 2012 IEEE Transactions on Antennas and Propagation 605890

[3] Bird D 1994 Radar Conference, 1994., Record of the 1994 IEEE National, March 29-31, 1994, Atlanta, GA, USA, p. 74

[4] De Adana F S et al 2003 IEE Proceedings-Radar, Sonar and Navigation 150375

[5] Susetio A et al 2015 Antennas and Propagation (APCAP), 2015 IEEE 4th Asia-Pacific Conference, June 30 - July 3, 2015, Kuta, Indonesia, p. 568

[6] Yuan L M et al 2018 Chinese Physics B 2744101

[7] Xu Y G et al 2018 J. Phys. D: Appl. Phys. 51 065004

[8] Lee J S et al 2013 Antennas and Propagation Society International Symposium (APSURSI) 2013 IEEE, July 7-13, 2013, Orlando, FL, USA, p. 606 